\title{
Resonances, Metastable States and Exponential Decay Laws in Perturbation Theory
}

\author{
Walter Hunziker \\ Institut für Theoretische Physik, ETH Zürich, Switzerland
}

Dedicated to Res Jost and Arthur Wightman

\begin{abstract}
Resonances which appear as perturbed bound states are discussed in the framework of Balslev-Combes theory. The corresponding metastable states are constructed using the formal perturbation expansion to order $N-1$ for the (nonexistent) perturbed bound states. They are shown to have exponential decay in time governed by the complex resonance energies, up to a background of order $2 N$ in the perturbation parameter. The results apply in lowest order $N=1$ to the perturbation of bound states embedded in the continuum and in arbitrary order to cases like the Stark effect.
\end{abstract}

\section{Introduction}

According to standard textbook wisdom, resonances of quantum systems correspond to metastable states which show exponential decay. The difficulties in making this statement precise are notorious and well explained in [13]. While Gamov's one-particle $\alpha$-decay model still receives attention (see e.g. $[16,17]$ ), relatively little is known in more general situations. In many cases the BalslevCombes theory of dilation analytic systems $[1,11]$ or one of its variants $[3,7,14,15]$ allows an elegant definition of the complex resonance energies, but there is yet no general description of the corresponding metastable states and their time evolution. Notable progress has recently been made by Orth [10], who developed a theory of resonances for $N$-body Schrödinger operators based on the Mourre estimate rather than dilation analyticity. The scope of our present contribution is more limited. We will use dilation analyticity and perturbation theory in the spirit of Simon [12] to discuss resonances which appear as perturbed bound states. By the same approach we can cover cases like the $N$-body Stark effect, where some results on exponential decay have previously been given by Herbst [4].

In the Balslev-Combes theory, the resonance energies are the complex eigenvalues of a "dilated" Hamiltonian $H(\Theta)$. For a typical $N$-body system, $H(\Theta)$ has a spectrum of the form shown in Fig. 1. To understand exponential decay in this setting we must answer the following questions. What is - in good approximation - 
a metastable state $\psi$ associated with a given resonance $\lambda$ ? Is there a time interval where

$$
\left(\psi, e^{-i H t} \psi\right) \approx e^{-i \lambda t}
$$

up to a relatively small background term?

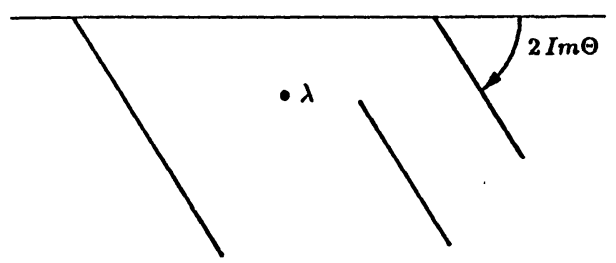

Fig. 1

Of course we would like to answer these questions simply in terms of the spectrum shown in Fig. 1, assuming that $\operatorname{Im} \lambda$ is small compared to the separation of $\lambda$ from the rest of the spectrum. This seems to be impossible - mainly because there is no general effective estimate for the resolvent $(z-H(\Theta))^{-1}$ in terms of the location of $z$ relative to the spectrum. For this reason we turn to the simpler case of a perturbed Hamiltonian

dilated into

$$
H_{\kappa}=H_{0}+\kappa V
$$

$$
H_{\kappa}(\Theta)=H_{0}(\Theta)+\kappa V(\Theta),
$$

and we only discuss resonances $\lambda_{\boldsymbol{\kappa}}$ which result from the perturbation of a (real) eigenvalue $\lambda_{0}$ of $H_{0}$.

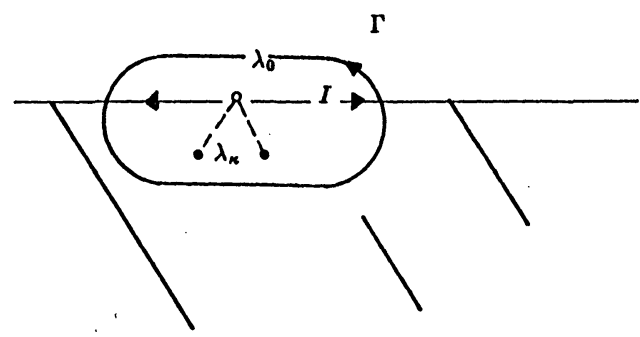

Fig. 2

The unperturbed bound states $\psi_{0}$ corresponding to the eigenvalue $\lambda_{0}$ are then obvious candidates for metastable states, and we can measure the background term in powers of the small perturbation parameter $\kappa$. As a typical result we will prove

$$
\left(\psi_{0}, e^{-i H_{\kappa} t} \psi_{0}\right)=e^{-i \lambda_{\kappa} t}+O\left(\kappa^{2}\right)
$$

in the nondegenerate case, uniformly in $0 \leqq t<\infty$. Our next question is whether this lowest order approximation for metastable states can be systematically improved. More precisely: is there an expansion

$$
\psi_{\kappa}^{N}=\psi_{0}+\kappa \psi_{1}+\ldots+\kappa^{N-1} \psi_{N-1}
$$


for metastable states which reduces the background term to higher order in $\kappa$ ? A natural candidate for (4) is the formal Rayleigh-Schrödinger (RS-) perturbation expansion for the (nonexistent) perturbed bound states. In fact, if the terms in this expansion are well-defined, we can improve (3) to

$$
\left(\psi_{\kappa}^{N}, e^{-i t H_{\kappa}} \psi_{\kappa}^{N}\right)=e^{-i \lambda_{\kappa} t}+O\left(\kappa^{2 N}\right) .
$$

In this way we obtain a physical interpretation of the RS-expansion for the "perturbed bound states" even in cases where no such states exist. We must emphasize, however, that this simple scheme works only in cases like the Stark effect, where $\lambda_{0}$ is a discrete eigenvalue of $H_{0}$. For embedded bound states the higher order terms in the RS-expansion (4) are not defined in Hilbert space, so that additional approximations (like the truncation of Gamov functions) must be used to construct metastable states. We will not investigate this possibility here.

\section{Preliminaries}

In this section we define the framework used throughout this paper.

(a) Balslev-Combes Theory. $H_{\kappa}$ is a family of selfadjoint operators defined for small $\kappa \geqq 0$. $U(\Theta)$ is a strongly continuous one parameter unitary group such that for fixed $\kappa$

$$
H_{\kappa}(\Theta)=U(\Theta) H_{\kappa} U(\Theta)^{-1}
$$

extends from real $\Theta$ to an analytic family in a strip $|\operatorname{Im} \Theta|<\beta$. (In $H_{\kappa}$ and elsewhere we simply drop the variable $\Theta$ to indicate that $\Theta$ is set equal to zero.) The spectrum of $H_{\kappa}(\Theta)$ depends only on $\operatorname{Im} \Theta$ and is assumed to lie in the closed lower halfplane for $\operatorname{Im} \Theta>0$. The relation

$$
H_{\kappa}(\Theta)^{*}=H_{\kappa}(\bar{\Theta})
$$

holds for real $\Theta$ and extends by analyticity to $|\operatorname{Im} \Theta|<\beta . U(\Theta)$ itself is defined for complex $\Theta$ by the spectral representation

$$
U(\Theta)=\int e^{-i s \Theta} d E(s)
$$

on the natural domain, satisfying $U(\Theta)^{*}=U(\bar{\Theta})^{-1}$. [We recall that $U(\Theta)$ must be unbounded for $\operatorname{Im} \Theta \neq 0$ if the spectrum of $H_{0}(\Theta)$ is to depend nontrivially on $\Theta$.] $\lambda_{0}$ is an isolated or embedded eigenvalue of $H_{0}$ with eigenprojection $P_{0}$, $\operatorname{dim} P_{0}=m_{0}<\infty$. We assume that $\lambda_{0}$ is separated from the essential spectrum of $H_{0}(\Theta)$ for $\operatorname{Im} \Theta \neq 0$. Then $\lambda_{0}$ is a discrete eigenvalue of $H_{0}(\Theta)$. Its eigenprojection $P_{0}(\Theta)$ is analytic in the full strip $|\operatorname{Im} \Theta|<\beta$. In particular,

$$
M_{0}(\Theta)=\operatorname{ran} P_{0}(\Theta)
$$

has constant dimension $m_{0}$. The relation

$$
P_{0}(\Theta)=U(\Theta) P_{0} U(\Theta)^{-1}
$$

holds on the dense domain of $U(\Theta)^{-1}$. Since $P_{0}$ has finite rank it follows that $M_{0}$ is in the domain of $U(\Theta)$ so that $U(\Theta)$ and $U(\Theta)^{-1}$ act as bounded operators from 
$M_{0}$ onto $M_{0}(\Theta)$ and vice-versa. The norms of these maps will in fact enter in our decay estimates. So far we have summarized the Balslev-Combes framework in abstract form.

(b) Stability. For fixed $\Theta$ with $\operatorname{Im} \Theta \neq 0$ we now consider the perturbation of the discrete eigenvalue $\lambda_{0}$ of $H_{0}(\Theta)$ by the family $H_{\kappa}(\Theta)$. First we require stability in the following sense [8]:

(i) There is a punctured neighbourhood $W(\Theta)$ of $\lambda_{0}$ (i.e. a complex neighbourhood with the point $\lambda_{0}$ removed) such that the resolvent $R_{\kappa}(\Theta, z)$ $=\left(z-H_{\kappa}(\Theta)\right)^{-1}$ exists and is uniformly bounded for each fixed $z \in W(\Theta)$ and $0 \leqq \kappa<\kappa_{0}(z)$.

(ii) The perturbed spectral projection

$$
P_{\kappa}(\Theta)=(2 \pi i)^{-1} \oint_{\Gamma} d z R_{\kappa}(\Theta, z)
$$

satisfies

$$
\lim _{\kappa \rightarrow 0}\left\|P_{\kappa}(\Theta)-P_{0}(\Theta)\right\|=0
$$

Here $\Gamma$ is an arbitrary loop in $W(\Theta)$ around $\lambda_{0}$. As a consequence of (i), $R_{\kappa}(\Theta, z)$ is uniformly bounded for $z \in \Gamma$ and $0 \leqq \kappa<\kappa_{0}(\Gamma)$. Equation (7) implies that $\operatorname{dim} P_{\kappa}(\Theta)=m_{0}$ for small $\kappa$. Therefore $\lambda_{0}$ is the limit as $\kappa \rightarrow 0$ of a group of perturbed eigenvalues $\lambda_{\kappa}$ having total algebraic multiplicity $m_{0}$. These are the eigenvalues of the reduced operator

$$
\tilde{H}_{\kappa}(\Theta)=P_{\kappa}(\Theta) H_{\kappa}(\Theta) P_{\kappa}(\Theta)
$$

acting on $M_{\kappa}(\Theta)=\operatorname{ran} P_{\kappa}(\Theta)$. In fact, the spectrum of $\tilde{H}_{\kappa}(\Theta)$ depends only on the sign of $\operatorname{Im} \Theta$, due to the relations

$$
\begin{aligned}
& P_{\kappa}\left(\Theta_{2}\right)=U\left(\Theta_{2}-\Theta_{1}\right) P_{\kappa}\left(\Theta_{1}\right) U\left(\Theta_{2}-\Theta_{1}\right)^{-1}, \\
& \tilde{H}_{\kappa}\left(\Theta_{2}\right)=U\left(\Theta_{2}-\Theta_{1}\right) \tilde{H}_{\kappa}\left(\Theta_{1}\right) U\left(\Theta_{2}-\Theta_{1}\right)^{-1},
\end{aligned}
$$

which hold for small $\kappa$ if $\operatorname{Im} \Theta_{1}$ and $\operatorname{Im} \Theta_{2}$ have the same sign. By convention we denote with $\lambda_{\kappa}$ the eigenvalues of $\tilde{H}_{\kappa}(\Theta)$ for $\operatorname{Im} \Theta>0$. These are the resonances corresponding to the unperturbed eigenvalue $\lambda_{0}$. Our assumption on the spectrum of $H_{\kappa}(\Theta)$ implies that $\operatorname{Im} \lambda_{\kappa} \leqq 0$.

(c) RS-Expansion. For the purpose of using RS-expansions we restrict $H_{\kappa}(\Theta)$ to the form (2). More precisely we assume that $V(\Theta)$ is given for $|\operatorname{Im} \Theta|<\beta$ as a densely defined, closed operator with $V(\Theta)^{*}=V(\bar{\Theta})$, such that $(2)$ holds on a core of $H_{\kappa}(\Theta)$. Then the iterated resolvent equation

$$
\begin{aligned}
R_{\kappa}(\Theta, z) P_{0}(\Theta)= & \sum_{m=0}^{N-1} \kappa^{m} R_{0}(\Theta, z) A_{m}(\Theta, z)+\kappa^{N} R_{\kappa}(\Theta, z) A_{N}(\Theta, z) ; \\
& A_{m}(\Theta, z)=\left[V(\Theta) R_{0}(\Theta, z)\right]^{m} P_{0}(\Theta)
\end{aligned}
$$

is valid for $\operatorname{Im} \Theta \neq 0, z \in W(\Theta)$ and small $\kappa$ as long as the individual terms are well defined (cf. [8], Lemma 8.1). We are interested in the case where the expanded part i.e. the terms not involving $R_{\kappa}(\Theta, z)$ - remains well-defined for $\operatorname{Im} \Theta=0$. Therefore we assume that the finite rank operators $A_{m}(\Theta, z)$ are analytic in $\Theta$ in the full strip 
$|\operatorname{Im} \Theta|<\beta$ for $m=1 \cdots N$ and all $z$ in some punctured neighbourhood $W$ of $\lambda_{0}$, with the properties

on $M_{0}(\Theta)$, and

$$
A_{m}(\Theta, z)=U(\Theta) A_{m}(z) U(\Theta)^{-1}
$$

$$
\sup _{z \in \Gamma}\left\|A_{m}(\Theta, z)\right\|<\infty
$$

for any loop $\Gamma \subset W$ around $\lambda_{0}$. The order $N \geqq 1$ will be specified in each case. For $N$ $=1$ our hypothesis only requires that $V(\Theta) P_{0}(\Theta)$ is analytic in $|\operatorname{Im} \Theta|<\beta$ with the transformation law (12). For $N>1$, however, it is implied that $R_{0}(\Theta, z)$ exists for $|\operatorname{Im} \Theta|<\beta$ and $z \in W$, ie. that $\lambda_{0}$ is a discrete eigenvalue of $H_{0}$.

Our list of assumptions is now complete. Typical examples are:

a) Analytic perturbations of embedded eigenvalues in dilation-analytic systems [12]. Here $V(\Theta)$ is bounded relative to $H_{0}(\Theta)$ so that our hypothesis is satisfied for $N=1$.

b) The Stark effect for discrete eigenvalues of Coulomb systems [5, 8]. This is an example where the formal RS-expansion for the (nonexistent) perturbed bound states is well-defined to any finite order, and where our assumptions are valid for arbitrary $N$.

At this point the reader may proceed directly to Sect. 3 . We continue with a discussion of the RS-expansion in the degenerate case as a preparation of Sect. 4. Inserting (10) into (6) we obtain the expansion of $P_{\kappa}(\Theta)$ for fixed $\Theta$ with $\operatorname{Im} \Theta \neq 0$ :

$$
P_{\kappa}(\Theta) P_{0}(\Theta)=B_{\kappa}(\Theta)+O\left(\kappa^{N}\right),
$$

where the expanded part $B_{\kappa}(\Theta)$ is analytic in the full strip $|\operatorname{Im} \Theta|<\beta$. Similar expansions result for

and for the operator

$$
P_{0}(\Theta) P_{\kappa}(\Theta)=\left[P_{\kappa}(\bar{\Theta}) P_{0}(\bar{\Theta})\right]^{*}
$$

$$
D_{\kappa}(\Theta)=P_{0}(\Theta) P_{\kappa}(\Theta) P_{0}(\Theta)
$$

acting on $M_{0}(\Theta)$. The RS-expansion of $P_{\kappa}(\Theta)$ is now obtained from the identity

$$
P_{\kappa}(\Theta)=P_{\kappa}(\Theta) P_{0}(\Theta) D_{\kappa}(\Theta)^{-1} P_{0}(\Theta) P_{\kappa}(\Theta),
$$

valid for small $\kappa$. To justify (15) we note that $D_{\kappa}(\Theta) \rightarrow 1$ as $\kappa \rightarrow 0$. Thus $D_{\kappa}(\Theta)^{-1 / 2} P_{0}(\Theta) P_{\kappa}(\Theta)$ is well-defined as an operator from $M_{\kappa}(\Theta)$ to $M_{0}(\Theta)$. By (14) it has a right inverse $P_{\kappa}(\Theta) P_{0}(\Theta) D_{\kappa}(\Theta)^{-1 / 2}$, and this is in fact the inverse since $\operatorname{dim} M_{\kappa}(\Theta)=\operatorname{dim} M_{0}(\Theta)$ for small $\kappa$. We write the RS-expansion of $P_{\kappa}(\Theta)$ as

$$
P_{\kappa}(\Theta)=P_{\kappa}^{N}(\Theta)+O\left(\kappa^{N}\right),
$$

noting that the expanded part $P_{\kappa}^{N}(\Theta)$ is analytic in $\Theta$ in the full strip $|\operatorname{Im} \Theta|<\beta$, satisfying

$$
P_{\kappa}^{N}(\Theta)=U(\Theta) P_{\kappa}^{N} U(\Theta)^{-1} .
$$

Here $P_{\kappa}^{N}=P_{\kappa}^{N}(0)$ is the formal perturbative expression to order $N-1$ of the "perturbed total eigenprojection of $H_{\kappa}$ " (which need not exist). Our aim is to derive decay estimates under $\exp \left(-i H_{\kappa} t\right)$ for the states in the space

$$
M_{\kappa}^{N}=\operatorname{ran}\left(P_{\kappa}^{N} P_{0}\right)
$$


which has dimension $m_{0}$ for small $\kappa$. This is prepared by the following construction. We define

$$
D_{\kappa}^{N}=U(\Theta)^{-1} P_{0}(\Theta) P_{\kappa}^{N}(\Theta) P_{\kappa}(\Theta) P_{\kappa}^{N}(\Theta) P_{0}(\Theta) U(\Theta)
$$

as an operator on $M_{0}$ for $\operatorname{Im} \Theta>0$, noting that the right-hand side depends only on the sign of $\operatorname{Im} \Theta$. For $\operatorname{Im} \Theta<0(17)$ remains valid if $D_{\kappa}^{N}$ is replaced by its adjoint. $D_{\kappa}^{N}$ has an expansion

$$
\begin{aligned}
& D_{\kappa}^{N}=d_{\kappa}^{N}+O\left(\kappa^{2 N}\right), \\
& d_{\kappa}^{N}=P_{0}\left(P_{\kappa}^{N}\right)^{2} P_{0}=\left(d_{\kappa}^{N}\right)^{*},
\end{aligned}
$$

which is derived from the identity

$$
\begin{aligned}
P_{\kappa}^{N}(\Theta) P_{\kappa}(\Theta) P_{\kappa}^{N}(\Theta)= & {\left[P_{\kappa}^{N}(\Theta)\right]^{2}+\left[P_{\kappa}^{N}(\Theta)-P_{\kappa}(\Theta)\right]\left[P_{\kappa}(\Theta)-1\right] } \\
& \cdot\left[P_{\kappa}^{N}(\Theta)-P_{\kappa}(\Theta)\right] .
\end{aligned}
$$

By the argument given to justify (15), we see that the map

$$
T_{\kappa}(\Theta)=P_{\kappa}(\Theta) P_{\kappa}^{N}(\Theta) P_{0}(\Theta) U(\Theta)\left(D_{\kappa}^{N}\right)^{-1 / 2}: M_{0} \rightarrow M_{\kappa}(\Theta)
$$

has the inverse

$$
T_{\kappa}(\Theta)^{-1}=\left(D_{\kappa}^{N}\right)^{-1 / 2} U(\Theta)^{-1} P_{0}(\Theta) P_{\kappa}^{N}(\Theta) P_{\kappa}(\Theta): M_{\kappa}(\Theta) \rightarrow M_{0}
$$

for $\operatorname{Im} \Theta>0$ and small $\kappa$. This is used to transform $\tilde{H}_{\kappa}(\Theta)$ into the equivalent operator

$$
h_{\kappa}=T_{\kappa}(\Theta)^{-1} \tilde{H}_{\kappa}(\Theta) T_{\kappa}(\Theta)
$$

acting on $M_{0}$, which is independent of $\Theta$ for $\operatorname{Im} \Theta>0$. By construction, the eigenvalues of $h_{\kappa}$ are the resonances $\lambda_{\kappa}$. We will prove below that

on $M_{0}$, where

$$
h_{\kappa}=t_{\kappa}^{*} H_{\kappa} t_{\kappa}+O\left(\kappa^{2 N}\right),
$$

$$
t_{\kappa}=P_{\kappa}^{N} P_{0}\left(d_{\kappa}^{N}\right)^{-1 / 2}
$$

maps $M_{0}$ isometrically onto $M_{\kappa}^{N}$ for small $\kappa$. This shows in particular that

which gives the estimate

$$
h_{\kappa}^{*}-h_{\kappa}=O\left(\kappa^{2 N}\right) \text {, }
$$

$$
\operatorname{Im} \lambda_{\kappa}=O\left(\kappa^{2 N}\right)
$$

for the width of the resonances. To prove (22) we first remark that $M_{\kappa}^{N}(\Theta)$ $=\operatorname{ran} P_{\kappa}^{N}(\Theta) P_{0}(\Theta) \subset D\left(H_{\kappa}(\Theta)\right)$ for $|\operatorname{Im} \Theta|<\beta$. Indeed, $z-H_{\kappa}(\Theta)$ can be applied to each term in the expanded part of (10), with the result

$$
\left(z-H_{\kappa}(\Theta)\right) R_{0}(\Theta, z) A_{m}(\Theta, z)=A_{m}(\Theta, z)-\kappa A_{m+1}(\Theta, z)
$$

for $z \in W$ and $m=0 \cdots N-1$. From the remainder in (10) we also see that

$$
H_{\kappa}(\Theta)\left(P_{\kappa}^{N}(\Theta)-P_{\kappa}(\Theta)\right) P_{0}(\Theta)=O\left(\kappa^{N}\right)
$$

if $\operatorname{Im} \Theta \neq 0$. Now we write out (21) and use the decomposition

$$
\tilde{H}_{\kappa}(\Theta)=H_{\kappa}(\Theta)-\left(1-P_{\kappa}(\Theta)\right) H_{\kappa}(\Theta)\left(1-P_{\kappa}(\Theta)\right) \text {. }
$$


The contribution of the last term to $h_{\kappa}$ is of order $\kappa^{2 N}$, since

$$
H_{\kappa}(\Theta)\left(1-P_{\kappa}(\Theta)\right) P_{\kappa}^{N}(\Theta) P_{0}(\Theta)=\left(1-P_{\kappa}(\Theta)\right) H_{\kappa}(\Theta)\left(P_{\kappa}^{N}(\Theta)-P_{\kappa}(\Theta)\right) P_{0}(\Theta)
$$

and

$$
P_{\kappa}^{N}(\Theta)\left(1-P_{\kappa}(\Theta)\right)=\left(P_{\kappa}^{N}(\Theta)-P_{\kappa}(\Theta)\right)\left(1-P_{\kappa}(\Theta)\right)
$$

are both of order $\kappa^{N}$. Using (18) we thus find

$$
\begin{aligned}
h_{\kappa}= & \left(d_{\kappa}^{N}\right)^{-1 / 2} U(\Theta)^{-1} P_{0}(\Theta) P_{\kappa}^{N}(\Theta) H_{\kappa}(\Theta) P_{\kappa}^{N}(\Theta) P_{0}(\Theta) U(\Theta)\left(d_{\kappa}^{N}\right)^{-1 / 2} \\
& +O\left(\kappa^{2 N}\right)
\end{aligned}
$$

where we can now set $\Theta=0$.

\section{The Nondegenerate Case in Lowest Order}

In this section we discuss the simplest case $m_{0}=N=1$ to give the essence of the argument.

Theorem 1. Let $\lambda_{0}$ be a simple (discrete or embedded) eigenvalue of $H_{0}$ with normalized eigenvector $\psi_{0}$. Let $g \in C_{0}^{\infty}(R)$ be supported sufficiently close to $\lambda_{0}$ with $g=1$ in some open interval containing $\lambda_{0}$. Then

$$
\left(\psi_{0}, e^{-i H_{\kappa} t} g\left(H_{\kappa}\right) \psi_{0}\right)=a(\kappa) e^{-i \lambda_{\kappa} t}+b(\kappa, t)
$$

for small $\kappa$ and $0 \leqq t<\infty$, where $\lambda_{\kappa}$ is the resonance eigenvalue and

$$
|b(\kappa, t)| \leqq \kappa^{2} c_{m}(1+t)^{-m}
$$

for any $m \geqq 0$. $a(\kappa)$ is given for (arbitrary) $\Theta$ in $0<\operatorname{Im} \Theta<\beta$ by

$$
\begin{aligned}
a(\kappa) & =\left(U(\bar{\Theta}) \psi_{0}, P_{\kappa}(\Theta) U(\Theta) \psi_{0}\right) \\
& =1+O\left(\kappa^{2}\right) .
\end{aligned}
$$

Proof. We fix $\Theta$ with $0<\operatorname{Im} \Theta<\beta$. By hypothesis there exists an open interval $I \ni \lambda_{0}$ with endpoints in the set $W(\Theta)$ defined in Sect. 2. Let $g \in C_{0}^{\infty}(I)$. Then

$$
\begin{aligned}
F(t) & =\left(\psi_{0}, e^{-i H_{\kappa} t} g\left(H_{\kappa}\right) \psi_{0}\right) \\
& =\lim _{\varepsilon}(2 \pi i)^{-1} \int_{I} d z e^{-i z t} g(z)\left(\psi_{0},\left[R_{\kappa}(z-i \varepsilon)-R_{\kappa}(z+i \varepsilon)\right] \psi_{0}\right) .
\end{aligned}
$$

This can be expressed in terms of $H_{\kappa}(\Theta)$ by

$$
\begin{aligned}
F(t) & =f(\bar{\Theta}, t)-f(\Theta, t), \\
f(\Theta, t) & =(2 \pi i)^{-1} \int_{I} d z e^{-i z t} g(z)\left(\psi_{0}(\bar{\Theta}), R_{\kappa}(\Theta, z) \psi_{0}(\Theta)\right),
\end{aligned}
$$

where $\psi_{0}(\Theta)=U(\Theta) \psi_{0}$. Here we have assumed for simplicity that $\operatorname{Im} \lambda_{\kappa}<0$ so that $I$ is contained in the resolvent set of $H_{\kappa}(\Theta)$ for small $\kappa$. If $\lambda_{\kappa}$ is real, then the integral (29) must be modified by a detour around $\lambda_{0}$ in the upper halfplane. This is of no consequence for our estimates and will be ignored. 
Next we choose a loop $\Gamma \subset W(\Theta)$ around $I$ as shown in Fig. 2. For $z$ inside $\Gamma$ we decompose the resolvent into singular and regular parts:

noting that

$$
\begin{aligned}
& R_{\kappa}(\Theta, z)=P_{\kappa}(\Theta)\left(z-\lambda_{\kappa}\right)^{-1}+\hat{R}_{\kappa}(\Theta, z), \\
& \hat{R}_{\kappa}(\Theta, z)=(2 \pi i)^{-1} \oint_{\Gamma} d \xi R_{\kappa}(\Theta, \xi)(\xi-z)^{-1},
\end{aligned}
$$

$$
\hat{R}_{\kappa}(\Theta, z) P_{\kappa}(\Theta)=P_{\kappa}(\Theta) \hat{R}_{\kappa}(\Theta, z)=0
$$

(a) Contribution of the regular part. Using (31) we can write the contribution of $\hat{R}_{\kappa}(\Theta, z)$ to $f(\Theta, t)$ as

where

$$
\left(u_{\kappa}(\bar{\Theta}),(2 \pi i)^{-1} \int_{I} d z e^{-i z t} g(z) \hat{R}_{\kappa}(\Theta, z) u_{\kappa}(\Theta)\right),
$$

$$
u_{\kappa}(\Theta)=\left[P_{0}(\Theta)-P_{\kappa}(\Theta)\right] \psi_{0}(\Theta)
$$

is of order $\kappa$. By partial integration the last integral is seen to be bounded by $a_{m} t^{-m}$ for any $m \geqq 0$ since $g \in C_{0}^{\infty}(I)$. The constants $a_{m}$ involve norms of derivatives (i.e. powers) of $\hat{R}_{\kappa}(\Theta, z)$. In fact $\hat{R}_{\kappa}(\Theta, z)$ is uniformly bounded for small $\kappa$ and $z \in I$ : this follows from (30) since $\Gamma \subset W(\Theta)$. As a result, the contribution of the regular part to $f(\Theta, t)$ is bounded by $\kappa^{2} c_{m}(1+t)^{-m}$ for small $\kappa$ and any $m \geqq 0$. The contribution to $f(\bar{\Theta}, t)$ is estimated in the same way.

(b) Contribution of the singular part. The singular part in (30) gives rise to the term

$$
\overline{a(\kappa)}(2 \pi i)^{-1} \int_{I} d z e^{-i z t} g(z)\left(z-\overline{\lambda_{\kappa}}\right)^{-1}-a(\kappa)(2 \pi i)^{-1} \int_{I} d z e^{-i z t} g(z)\left(z-\lambda_{\kappa}\right)^{-1}
$$

in $F(t)$, where $a(\kappa)$ is defined by (28). We now use the fact that $g=1$ on some open interval $I_{0} \ni \lambda_{0}$ to deform the path $I$ in both integrals to the lower halfplane, as shown in Fig. 3:

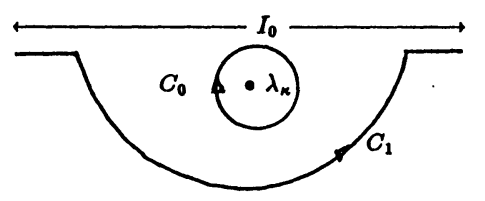

Fig. 3

From the second integral in (32) we pick up the residue $a(\kappa) e^{-i \lambda_{\kappa} t}$ at the point $z=\lambda_{\kappa}$. The remainder is given by (32) with both integrals taken along the path $C_{1}$ (where $g(z)=1$ for $\operatorname{Im} z<0$ ). Using the identity

$$
P_{0}(\Theta) P_{\kappa}(\Theta) P_{0}(\Theta)=\left[P_{0}(\Theta)\right]^{2}+\left[P_{0}(\Theta)-P_{\kappa}(\Theta)\right]\left[P_{\kappa}(\Theta)-1\right]\left[P_{0}(\Theta)-P_{\kappa}(\Theta)\right]
$$

and the fact that $\left(\psi_{0}(\bar{\Theta}), \psi_{0}(\Theta)\right)=\left(\psi_{0}, \psi_{0}\right)=1$, we see that $a(\kappa)=1+O\left(\kappa^{2}\right)$. Thus we can write the remainder in the form 


$$
\begin{aligned}
& \left(\operatorname{Im} \lambda_{\kappa}\right) \pi^{-1} \int_{C_{1}} d z e^{-i z t} g(z)\left(z-\overline{\lambda_{\kappa}}\right)^{-1}\left(z-\lambda_{\kappa}\right)^{-1} \\
& \quad+O\left(\kappa^{2}\right) \int_{C_{1}} d z e^{-i z t} g(z)\left(z-\overline{\lambda_{\kappa}}\right)^{-1}+O\left(\kappa^{2}\right) \int_{C_{1}} d z e^{-i z t} g(z)\left(z-\lambda_{\kappa}\right)^{-1} .
\end{aligned}
$$

For $0 \leqq t<\infty$ all three integrals have bounds of the form $a_{m} t^{-m}$ with arbitrary $m \geqq 0$. The proof is completed by noting that $\operatorname{Im} \lambda_{\kappa}=O\left(\kappa^{2}\right)$.

Discussion. With Theorem 1 we can construct the metastable states

$$
\phi_{\kappa}=g\left(H_{\kappa}\right) \psi_{0}\left\|g\left(H_{\kappa}\right) \psi_{0}\right\|^{-1} .
$$

Since $|g|^{2}$ also satisfies the conditions imposed on $g$ we obtain the decay estimate

$$
\left(\phi_{\kappa}, e^{-i H_{\kappa} t} \phi_{\kappa}\right)=(1-b(\kappa, 0)) e^{-i \lambda_{\kappa} t}+b(\kappa, t)
$$

with $|b(\kappa, t)| \leqq \kappa^{2} c_{m}(1+t)^{-m}$ for small $\kappa, 0 \leqq t<\infty$ and any $m \geqq 0$. To discuss the decay of $\psi_{0}$ itself we choose $0 \leqq g \leqq 1$. By Theorem 1 we have

$$
\left(\psi_{0}, e^{-i H_{\kappa} t} g\left(H_{\kappa}\right) \psi_{0}\right)=e^{-i \lambda_{\kappa} t}+O\left(\kappa^{2}\right)
$$

uniformly in $0 \leqq t<\infty$. Setting $t=0$ we find

$$
\left(\psi_{0},\left(1-g\left(H_{\kappa}\right)\right) \psi_{0}\right)=\left\|\left(1-g\left(H_{\kappa}\right)\right)^{1 / 2} \psi_{0}\right\|^{2}=O\left(\kappa^{2}\right) .
$$

Inserting this into (34) we arrive at the result

$$
\left(\psi_{0}, e^{-i H_{\kappa} t} \psi_{0}\right)=e^{-i \lambda_{\kappa} t}+O\left(\kappa^{2}\right) .
$$

\section{The General Case}

Here we extend the decay estimate to the general case within the framework of Sect. 2, where $m_{0}$ and $N$ are now arbitrary.

Theorem 2. If $g$ is chosen as in Theorem 1, then, as an operator relation on the unperturbed eigenspace $M_{0}$,

$$
P_{0} P_{\kappa}^{N} e^{-i H_{\kappa} t} g\left(H_{\kappa}\right) P_{\kappa}^{N} P_{0}=\left(D_{\kappa}^{N}\right)^{1 / 2} e^{-i h_{\kappa} t}\left(D_{\kappa}^{N}\right)^{1 / 2}+B(\kappa, t)
$$

for small $\kappa$ and $0 \leqq t<\infty$, where

$$
\|B(\kappa, t)\| \leqq \kappa^{2 N} c_{m}(1+t)^{-m}
$$

for any $m \geqq 0$ and corresponding constants $c_{m}$.

Proof. As in the proof of Theorem 1 we have

$$
\begin{aligned}
F(t)= & P_{0} P_{\kappa}^{N} e^{-i H_{\kappa} t} g\left(H_{\kappa}\right) P_{\kappa}^{N} P_{0} \\
= & f(\Theta, t)-f(\Theta, t), \\
f(\Theta, t)= & (2 \pi i)^{-1} \int_{I} d z e^{-i z t} g(z) U(\Theta)^{-1} P_{0}(\Theta) P_{\kappa}^{N}(\Theta) R_{\kappa}(\Theta, z) P_{\kappa}^{N}(\Theta) \\
& \cdot P_{0}(\Theta) U(\Theta),
\end{aligned}
$$


for $\operatorname{Im} \Theta>0$, where $U(\Theta)$ is the bounded operator $M_{0} \rightarrow M_{0}(\Theta)$. The resolvent $R_{\kappa}(\Theta, z)$ has the singular part

$$
P_{\kappa}(\Theta) R_{\kappa}(\Theta, z) P_{\kappa}(\Theta)
$$

and the regular part $\hat{R}_{\kappa}(\Theta, z)$ given by (30). The contribution of the regular part to $(F(t)$ is estimated as before, using that

$$
\begin{aligned}
P_{\kappa}^{N}(\Theta) \hat{R}_{\kappa}(\Theta, z) P_{\kappa}^{N}(\Theta) & =\left[P_{\kappa}^{N}(\Theta)-P_{\kappa}(\Theta)\right] \hat{R}_{\kappa}(\Theta, z)\left[P_{\kappa}^{N}(\Theta)-P_{\kappa}(\Theta)\right] \\
& =O\left(\kappa^{2 N}\right)
\end{aligned}
$$

As a result this contribution has a bound of the form (37) for any $m \geqq 0$. Using (21) we can write the contribution of the singular part (38) to $f(\Theta, t)$ as

$$
\left(D_{\kappa}^{N}\right)^{1 / 2}(2 \pi i)^{-1} \int_{I} d z e^{-i z t} g(z)\left(z-h_{\kappa}\right)^{-1}\left(D_{\kappa}^{N}\right)^{1 / 2} .
$$

The contribution to $f(\bar{\Theta}, t)$ has the same form, with the difference that $D_{\kappa}^{N}$ and $h_{\kappa}$ are replaced by their adjoints. In both terms we deform the path $I$ as shown in Fig. 3 , where the loop $C_{0}$ now encloses the full spectrum of $h_{\kappa}$, i.e. all the resonance eigenvalues $\lambda_{\kappa}$ corresponding to $\lambda_{0}$. This loop gives rise to the term

$$
\left(D_{\kappa}^{N}\right)^{1 / 2} e^{-i h_{\kappa} t}\left(D_{\kappa}^{N}\right)^{1 / 2}
$$

in $F(t)$, which comes from $f(\Theta, t)$. The remainder is given by

$$
\begin{gathered}
\left(D_{\kappa}^{N *}\right)^{1 / 2}(2 \pi i)^{-1} \int_{C_{1}} d z e^{-i z t} g(z)\left(z-h_{\kappa}^{*}\right)^{-1}\left(D_{\kappa}^{N *}\right)^{1 / 2} \\
-\left(D_{\kappa}^{N}\right)^{1 / 2}(2 \pi i)^{-1} \int_{C_{1}} d z e^{-i z t} g(z)\left(z-h_{\kappa}\right)^{-1}\left(D_{\kappa}^{N}\right)^{1 / 2} .
\end{gathered}
$$

Using (18) we can replace $D_{\kappa}^{N}$ and its adjoint by $d_{\kappa}^{N}$-committing an error of the form (37). Then we combine the two terms of (39) to

$$
\left(d_{\kappa}^{N}\right)^{1 / 2}(2 \pi i)^{-1} \int_{C_{1}} d z e^{-i z t} g(z)\left(z-h_{\kappa}^{*}\right)^{-1}\left(h_{\kappa}^{*}-h_{\kappa}\right)\left(z-h_{\kappa}\right)^{-1}\left(d_{\kappa}^{N}\right)^{1 / 2}
$$

which by (24) has again a bound of the form (37).

Discussion. Metastable states with exponential decay laws are obtained from the eigenvectors of $h_{\kappa}$. Let

$$
\phi_{\kappa}=g\left(H_{\kappa}\right) P_{\kappa}^{N}\left(D_{\kappa}^{N}\right)^{-1 / 2} \psi_{\kappa}
$$

be normalized to 1 , with $h_{\kappa} \psi_{\kappa}=\lambda_{\kappa} \psi_{\kappa}$. Then

$$
\left(\phi_{\kappa}, e^{-i H_{\kappa} t} \phi_{\kappa}\right)=(1-b(\kappa, 0)) e^{-i \lambda_{\kappa} t}+b(\kappa, t)
$$

for small $\kappa$ and $0 \leqq t<\infty$, where

$$
|b(\kappa, t)| \leqq \kappa^{2 N} c_{m}(1+t)^{-m}
$$

for any $m \geqq 0$. In the example of the Stark effect $N$ is arbitrary and $h_{\kappa}$ has a complete 
set of eigenvectors [6]. In general, the Jordan normal form of $h_{\kappa}$ must be used to exhibit the $t$-dependence of $\exp \left(-i h_{\kappa} t\right)$. In analogy to (35) we can also find decay laws for the states in $M_{\kappa}^{N}$ with a time-independent background estimate. From (36) and (18) we obtain

$$
t_{\kappa}^{*} e^{-i H_{\kappa} t} g\left(H_{\kappa}\right) t_{\kappa}=e^{-i h_{\kappa} t}+O\left(\kappa^{2 N}\right),
$$

where $t_{\kappa}$ is given by (23). Choosing $0 \leqq g \leqq 1$ and setting $t=0$ we find

$$
\left\|\left(1-g\left(H_{\kappa}\right)\right)^{1 / 2} t_{\kappa}\right\|^{2}=O\left(\kappa^{2 N}\right)
$$

and therefore

$$
\left(t_{\kappa} \phi, e^{-i H_{\kappa} t} t_{\kappa} \psi\right)=\left(\phi, e^{-i h_{\kappa} t} \psi\right)+O\left(\kappa^{2 N}\right)
$$

uniformly for states $\phi, \psi \in M_{0}$ and $0 \leqq t<\infty$. In the nondegenerate case this is the result quoted in (5): then $t_{\kappa} \psi$ is the formal "perturbed eigenstate to order $N-1$ ". As a consequence of (41) we also note that $\left\|\exp \left(-i h_{\kappa} t\right)\right\| \leqq 1+O\left(\kappa^{2 N}\right)$. In fact we see no reason to believe that $\exp \left(-i h_{\kappa} t\right)$ should be a contraction.

Acknowledgement. I am indebted to J.M. Graf for pointing out an essential error in the first version of this paper.

\section{References}

1. Balslev, E., Combes, J.M.: Spectral properties of Schrödinger operators with dilation analytic interactions. Commun. Math. Phys. 22, 280-294 (1971)

2. Cycon, H.L., Froese, R.G., Kirsch, W., Simon, B.: Schrödinger operators. Berlin, Heidelberg, New York: Springer 1987

3. Cycon, H.L. : Resonances defined by modified dilations. Helv. Phys. Acta 58, 969-981 (1985)

4. Herbst, I.: Exponential decay in the Stark effect. Commun. Math. Phys. 87, 429-447 $(1982 / 83)$

5. Herbst, I., Simon, B. : Dilation analyticity in constant electric field. II. The N-body problem, Borel summability. Commun. Math. Phys. 80, 181-216 (1981)

6. Hunziker, W., Pillet, C.-A.: Degenerate asymptotic perturbation theory. Commun. Math. Phys. 90, 219-233 (1983)

7. Hunziker, W.: Distortion analyticity and molecular resonance curves. Ann. Inst. Henri Poincaré 45, 339-358 (1986)

8. Hunziker, W.: Notes on asymptotic perturbation theory for Schrödinger eigenvalue problems. Helv. Phys. Acta 61, 257-304 (1988)

9. Kato, T.: Perturbation theory for linear operators. Berlin, Heidelberg, New York: Springer 1966

10. Orth, A.: Quantum mechanical resonance and limiting absorption: the many body problem. Commun. Math. Phys. 126, 559-573 (1990)

11. Reed, M., Simon, B.: Methods of modern mathematical physics. IV. Analysis of operators. New York: Academic Press 1978

12. Simon, B. : Resonances in $N$-body quantum systems with dilation analytic potentials and the foundations of time-dependent perturbation theory. Ann. Math. 97, 247-274 (1973)

13. Simon, B.: Resonances and complex scaling: a rigorous overview. Int. J. Quant. Chem. 14, 529-542 (1978) 
14. Simon, B.: The definition of molecular resonance curves by the method of exterior complex scaling. Phys. Lett. 71 A, 211-214 (1979)

15. Sigal, I.M.: Complex transformation method and resonances in one-body quantum systems. Ann. Inst. Henri Poincaré 41, 103-114 (1984)

16. Skibsted, E.: Truncated Gamov functions, $\alpha$-decay and the exponential law. Commun. Math. Phys. 104, 591-604 (1986)

17. Skibsted, E.: On the evolution of resonance states. J. Math. Anal. Appl. (to appear)

Communicated by A. Jaffe

Received April 3, 1990 